groups, which indicated a dissociation of effects on biochemical and bone-density end points. Although the researchers found no significant effects of calcium supplementation on fractures, falls or vascular events, they urge confirmation of these findings.

The authors conclude that daily calcium supplementation of $1,200 \mathrm{mg}$ benefits bone health in healthy older men.

Original article Reid IR et al. (2008) Randomized controlled trial of calcium supplementation in healthy, nonosteoporotic, older men. Arch Intern Med 168: 2276-2282

\section{Aromatase inhibition restores testosterone levels in hypogonadal older men}

Aromatase inhibition could be an alternative to testosterone administration to mitigate declining androgen production in hypogonadal older men. Unlike testosterone replacement, aromatase inhibition lowers circulating estradiol levels, which potentially prevents adverse effects of testosterone supplementation. Burnett-Bowie et al. investigated the efficacy and safety of the synthetic aromatase inhibitor anastrozole to treat hypogonadism in aging men.

The study enrolled 88 men (60 years or older) with symptoms of hypogonadism and low testosterone levels $(5.2-10.4 \mathrm{nmol} / \mathrm{l}$ in a single measure or $10.4-12.1 \mathrm{nmol} / \mathrm{l}$ in two consecutive measures). Participants were randomly assigned to receive either anastrozole $1 \mathrm{mg}$ daily or placebo for 12 months; 11 men from the anastrozole group and 8 from the placebo withdrew from the study. Changes in gonadal steroid hormone levels, lower extremity strength, body composition (fat and muscle), lipid levels, prostate-specific antigen levels, and symptoms of urinary obstruction were all recorded at baseline and every 3 months thereafter.

Compared with placebo and baseline measurements, aromatase inhibition increased mean serum testosterone levels by $\sim 50 \%$ at 3 months. However, mean androgen levels decreased between months 3 and 12 (but remained significantly higher than baseline), which indicated an acquired resistance to anastrozole. Conversely, anastrozole therapy lowered estradiol levels, which did not revert to baseline between months 3 and 12; no such change was observed in the placebo group.

Aromatase inhibition improved testosterone levels to the mid-normal range and did not alter prostate-specific antigen levels or urinaryobstructive symptoms, but failed to improve body composition or strength.

Original article Burnett-Bowie SA et al. (2008) Effects

of aromatase inhibition in hypogonadal older men: a randomized, double-blind, placebo-controlled trial.

Clin Endocrinol (Oxf) [doi:10.1111/j.1365-2265.2008.03327.x]

\section{Abdominal adiposity is associated with risk of death in a large, European cohort}

Large studies of the association between abdominal adiposity and risk of death are scarce, despite the fact that abdominal adiposity is more closely related to the risk of certain chronic diseases than is BMI. In a large, European, multicenter study, Pischon et al. assessed the association of BMI, waist circumference and waist-to-hip ratio with risk of death.

The study included 359,387 participants of the Prospective Investigation into Cancer and Nutrition. Participants did not have a history of cancer, heart disease or stroke and had a mean age of 51.5 years at baseline. During follow-up (mean 9.7 years), 14,723 participants died. The association of BMI with risk of death was $J$-shaped, such that the lowest risk of death was observed in men and women with a BMl of $25.3 \mathrm{~kg} / \mathrm{m}^{2}$ and $24.3 \mathrm{~kg} / \mathrm{m}^{2}$, respectively. Even after adjustment for BMI, both waist circumference and waist-to-hip ratio were strongly associated with risk of death. Interestingly, associations between the two measures of abdominal adiposity and risk of death were stronger in participants with a lower BMI than in those with a higher BMI.

The findings support the use of waist circumference or waist-to-hip ratio measurements, in addition to BMI, to assess patients' risk of death. These additional assessments may be particularly useful for patients of normal weight who, nonetheless, might have an increased risk of death owing to increased abdominal adiposity.

Original article Pischon T et al. (2008) General and abdominal adiposity and risk of death in Europe. N Engl J Med 359: $2105-2120$ 